\title{
HIV Disclosure, Condom Use, and Awareness of HIV Infection Among HIV-Positive, Heterosexual Drug Injectors in St. Petersburg, Russian Federation
}

\author{
Lauretta E. Grau Edward White $\cdot$ Linda M. Niccolai • \\ Olga V. Toussova $\cdot$ Sergei V. Verevochkin $\cdot$ \\ Andrei P. Kozlov $\cdot$ Robert Heimer
}

Published online: 11 August 2010

(C) The Author(s) 2010. This article is published with open access at Springerlink.com

\begin{abstract}
We examined the prevalence of HIV disclosure to sexual partners by HIV-positive drug injectors (IDUs) in St. Petersburg, Russia and compared the magnitude and direction of associations of condom use with awareness of one's HIV infection and disclosure to partners. Among 157 HIV-infected participants, awareness of infection at time of last intercourse was associated with condom use with partners perceived to be HIV-negative (aOR 6.68, 95\% CI 1.60-27.88). Among the 70 participants aware of their infection prior to enrolment, disclosure to potentially uninfected sexual partners was independently and negatively associated with condom use (aOR 0.13, 95\% CI $0.02-0.66)$. Disclosure was independently associated with having injected $\geq 9$ years (aOR 6.04, 95\% CI 1.53-23.77) and partnership with another IDU (aOR 3.61, 95\% CI 1.44-9.06) or HIV-seropositive (aOR 45.12, 95\% CI 2.79-730.46). Scaling up HIV testing services and interventions that increase the likelihood of individuals receiving their test results is recommended.
\end{abstract}

Keywords HIV disclosure - HIV testing - Condom use · Injection drug use $\cdot$ Russia

L. E. Grau $(\bowtie) \cdot$ E. White $\cdot$ L. M. Niccolai $\cdot$ R. Heimer Division of Epidemiology of Microbial Diseases, Yale University School of Public Health, 60 College Street, P.O. Box 208034, New Haven, CT 06520-8034, USA e-mail: lauretta.grau@yale.edu

O. V. Toussova $\cdot$ S. V. Verevochkin $\cdot$ A. P. Kozlov

The Biomedical Center, St. Petersburg, Russia

\section{Introduction}

Russia faces one of the most rapidly expanding AIDS epidemics [1,2], with more than 470,000 registered HIV cases at the end of 2008 and the estimated number of infections exceeding one million [3, 4]. Thus far, the overwhelming majority of those infected are injection drug users (IDUs) [1, 2, 5-8]. Estimates of HIV seroprevalence among St. Petersburg IDUs indicate a rise from $2-4 \%$ in 1998 to $11-18 \%$ in 2000 to $30 \%$ in 2002 to $49 \%$ in 2008 [9-14]. There is also evidence that HIV is spreading to the general population via heterosexual contact [7, 15-17].

In the West and particularly in the US, recent HIV prevention efforts have focused on promoting HIV counseling and testing with disclosure of HIV serostatus to prospective sexual partners [18, 19]. Estimates of the proportions of HIV-infected heterosexuals in the West who disclose to partners range from 60 to $80 \%$ [20-22]. By contrast, the issue of disclosure and its potential public health impact is largely unexplored in Russia. Only limited anonymous HIV testing is available in Russia, and lack of access to treatment as well as the pervasive stigma associated with HIV disease [23, 24] often dissuade people from being tested. Although many Russians have been tested for HIV at least once in their lives, the testing most commonly occurs as a result of incarceration or an inpatient medical visit. Only approximately one in five IDUs who self-identify as HIV-negative had been tested within the previous year [25]. Since HIV testing is not as prevalent as in the West, the relationship between awareness of one's HIV status, disclosure, and condom use is poorly understood. The current study is the first to examine these issues within a sample of IDUs in St. Petersburg, Russia.

In several US studies, disclosure appears to be influenced by contextual, temporal, and situational factors [26, 
27]. Individuals disclose to primary more than to non-primary or anonymous partners [22, 26-33]. Studies suggest that the spread of HIV may be fueled by a relatively small number of infected individuals who engage in multiple high-risk behaviors (e.g., multiple partners, unprotected sex) without disclosure [27, 34-36]. Partner characteristics also influence disclosure behavior such that disclosure was less likely to occur with younger partners [37] or with those whose serostatus is understood to be negative or to be unknown [28, 38-40]. Additionally, timing of disclosure (i.e., prior or subsequent to unprotected sex) is not clearly identified [27], and therefore, sex partners may underestimate their HIV risk [38, 41, 42]. People need time to adjust to their diagnosis-typically $2-3$ years-after which time disclosure is more likely to occur [39, 43-45].

The few studies assessing HIV testing awareness in Russia or other countries of the former Soviet Union suggest that fewer than $40 \%$ of Russian participants were aware of their partner's HIV status at time of sex [25, 46]. Pervasive stigma surrounding HIV in Russia [23, 24] may dissuade people from disclosing [47]. We are unaware of any scientific publications that specifically examine HIV disclosure behavior among Russian IDUs, although there is some evidence from Western Europe that IDUs' awareness of their HIV infection was associated with increased condom use [48]. Since the epidemic is currently concentrated among IDUs, we begin such explorations by seeking to identify individual and interpersonal factors that influence disclosure and condom use behaviors in this population. Specifically, we used data on recent sexual partnerships from HIV-infected IDUs, only some of whom had been aware of their infection at time of last intercourse to address four questions: (1) Among HIV-infected IDUs, is knowledge of their infection associated with increased condom use? (2) What individual, partner, and partnership characteristics are associated with disclosure by HIVpositive IDUs? (3) Is disclosure by HIV-positive IDUs associated with condom use? and (4) Is disclosure of positive status or awareness of positive status more strongly associated with condom use? This constitutes a first step in determining whether and the extent to which HIV disclosure should be emphasized in future prevention interventions for Russian IDUs.

\section{Methods}

As part of a larger multi-site, cross-sectional study, "Sexual Acquisition and Transmission of HIV - Cooperative Agreement Program" (SATH-CAP), data were collected in St. Petersburg from September 2005 to December 2008. SATH-CAP seeks to identify the structural, social, and individual factors associated with HIV risk behavior among high-risk populations, one of which is users of heroin, cocaine, or amphetamine-type stimulants, and the sex partners of these participants. Sampling and recruitment procedures, data collection instruments, and other study methods have been reported in detail elsewhere [25, 49]; specifics relevant to this study are described below.

\section{Participants}

Participants were recruited using a modified version of respondent-driven sampling (RDS) [49-51]. Previous analysis found less than 5\% overlap between drug users and men who have sex with men (MSM) and greater than $95 \%$ prevalence of injection among the drug users [10, 25]. The present analysis is restricted to SATH-CAP participants who (1) were 18 years or older, (2) reported injecting illicit drugs at least once in their life, (3) tested positive for HIV infection as part of the SATH-CAP serological testing, and (4) supplied information about their sex partners within the past 6 months (Fig. 1). Participants were provided incentives equivalent to US\$10 for their participation and US $\$ 5$ for each person they successfully referred to the study. All study and consent procedures and study

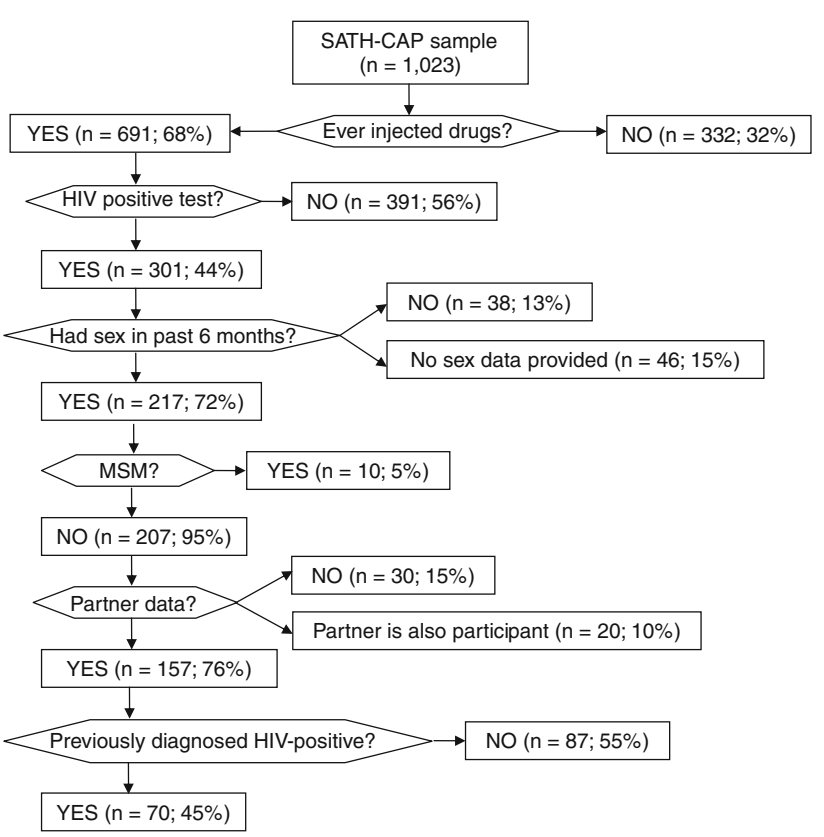

Fig. 1 Derivation from the SATH-CAP cohort of the subsample used in the current analyses. We limited the analyses to those participants who reported ever having injected drugs and who tested positive for HIV infection at enrolment $(n=300)$. We first examined the association between prior knowledge of HIV status and condom use at last intercourse in each of their sexual partnerships. Subsequently, among participants who were aware of their HIV infection prior to enrolment, we examined the association between disclosure of HIV status to and condom use with their reported sex partners 
measures were approved by institutional review boards at The Biomedical Center, Yale University, and RAND Corporation.

\section{Data Collection, Measures, and Laboratory Procedures}

Participants completed a structured, computer-assisted, self-administered interview and, following pre-test HIV counseling, had blood drawn for serological testing. Data used in the present analyses included demographic, medical history, drug and sex behaviors, sex partner information, and HIV serology. Demographic variables included sex, age, marital status, living situation, educational level, current employment status, legal income (past 30 days), and history of incarceration. Medical history items included HIV testing history and whether participants had received their most recent test results. Participants reporting HIV infection were asked the month and year of their diagnosis. We define "HIV-seropositive" as seropositive test results based on the testing performed as part of this study and "previously aware of diagnosis" as participants who reported having been informed that they were HIVpositive prior to enrolling in this study. Drug use data included duration of injection drug use (median-split at 9 years), number of injections in past 30 days (mediansplit at 22), and unsafe injections in past 30 days (yes/no; defined as sharing needles without using bleach, using a single syringe to mix or divide drugs, or sharing cookers, cotton, or water). Sexual behavior data included number of sex partners in the prior 6 months, number of new partners in the past 6 months, partner-specific information (i.e., for main partner, up to three most recent non-main partners, and if applicable, a partner with whom they also shared drugs), and condom use at last sexual intercourse with up to five partners in the previous 6 months. Partner-specific data included each partner's age and gender, partnership type (main vs. casual/friend vs. stranger/sex trade), disclosure of HIV status to partner, and perceived injection drug use history of partner. Perceived HIV status was ascertained by asking about each partner "What is [the partner's] HIV status?" and were given as potential responses "I don't know or am not sure", "I am sure he/she is HIV negative" and "I am sure he/she is HIV-positive". Participants who responded that a partner was positive were then asked "Do you know [the partner's] HIV status because he/she told you him/herself?" Participants were also asked for each partner "Does [the partner] know your HIV status?" Those answering in the affirmative were then asked "Does [the partner] know your HIV status because you told him/her yourself?" We included all reported partnerships in these analyses except those in the previously aware of diagnosis group for whom the date of last intercourse occurred before they became aware of their HIV diagnosis.
Analytical Methods

Standard descriptive statistics were used to describe the sample. Continuous variables were dichotomized in order to adapt those with non-normal distributions to parametric tests and to preserve statistical power. Using Chi-square and Wilcoxon rank-sum tests, we compared the characteristics of seropositives who were aware of their HIV infection with those who were not, examined whether the demographic characteristics of participants who provided information on sexual partnerships differed from those who did not, and examined whether partner characteristics were associated with the participant's perception of their partners' serostatus. We employed population-averaged panel-data models to account for single participants' ability to report on more than one partner. This was accomplished using generalized estimating equations (GEE), under the command-gee-in Stata 10 [52]. We used the same technique to assess among participants who were aware of their infection, whether any participant, partner, or partnership characteristic was associated with disclosure within the partnership.

Among partnerships reported by HIV-infected participants, we assessed bivariate associations between the participant's awareness of being infected with HIV at the time of their last sexual encounter and condom use at that time. We assessed these associations within all reported partnerships and within a subset of partnerships with partners either perceived to be uninfected or of unknown serostatus. To estimate associations between awareness of infection and condom use, unconfounded by other available individual, partner, and partnership characteristics, we also assessed associations between condom use and participant's age, duration of injection drug use, history of incarceration, partnership type, and participant's understanding of whether partners also injected drugs and perceived serostatus of partners. In each case, covariates that were associated in bivariate analyses at $P<0.20$ were entered into an initial multivariate model; backward selection eliminated covariates that did not remain significant at $P<0.05$ or did not change other coefficients by $>10 \%$. This was done for all partnerships and the three aforementioned subsets.

We then limited analysis to participants who were aware of their HIV infection prior to study enrolment. Among partnerships reported by these participants, we assessed bivariate associations between participants' disclosure of positive serostatus and condom use, and produced multivariate models in the manner described above for all reported partnerships and the three aforementioned subsets.

To account for potential confounders in any association between prior awareness of HIV infection and condom use, we identified associations between prior diagnosis of HIV infection and participant characteristics using Chi-square 
or Fisher's exact test; for non-normally distributed variables we used the Wilcoxon rank-sum test. For condom use, we calculated unadjusted and adjusted correlations between dependent and independent variables. In each case, covariates that were associated in bivariate analyses at $P<0.20$ were entered into an initial multivariate model; backward selection eliminated covariates that did not remain significant at $P<0.05$ or did not change other coefficients by $>10 \%$.

Participants could refer their sex partners to the study, raising the possibility of double-reporting of sex partnerships between two participants, once by the recruiter and once by the recruit. To account for potential correlation we nested responses by the participant who had referred the partner to the study. Therefore, partnerships reported with participants' recruiters were excluded from analysis if both participant and recruiter were present in the subset of observations analyzed.

Because participants were recruited using RDS, use of weighting procedures was considered to adjust the sample for recruitment probabilities to obtain estimates that would reflect the underlying population from which sampling was conducted. However, in prior analyses of these data, proportions of demographic and serologic characteristics did not differ substantially between weighted and unweighted distributions. Therefore, we used unweighted estimates for these analyses [25].

\section{Results}

Characteristics of the Study Sample

Of 691 participants who reported a history of drug injection, $301(44 \%)$ tested HIV-seropositive at the time of enrolment. Among these seropositive participants, 38 reported having no sex partners, 46 participants provided no data on the number of sex partners they had in the prior 6 months (therefore not asked partner-specific items), and 30 declined to provide partnership data. We also excluded from analysis ten males who reported sex with male partners only and 20 participants who reported sex partnership only with their recruiter. Thus, we restricted analyses to the 157 heterosexual, HIV-seropositive participants who provided data on 231 non-overlapping partnerships (Fig. 1). We found no significant demographic differences between participants who provided data partnership data and those who did not.

Nearly two-thirds of the resulting sample were male $(65 \%)$. The median age was 28 years $(25 \%, 75 \%=24$, 32 years), with approximately twice as many single participants as married/partnered participants. Most participants $(69 \%)$ lived with either their families or friends. Over half $(56 \%)$ had at least some post-secondary education. Few $(37 \%)$ were currently employed; median income from legal sources was 1,625 rubles (US\$50) in the last month $(25 \%, 75 \%=0,7,000$ rubles; data not shown). Fewer than half the participants (42\%) reported ever having been incarcerated. The median duration of injection drug use was 9.2 years $(25 \%, 75 \%=6,11$; data not shown), and nearly all (92\%) reported injecting within the prior 30 days. The median number of injections in the prior 30 days was $23(25 \%, 75 \%=10,30$; data not shown); the majority of participants $(79 \%)$ reported injecting unsafely (e.g., sharing syringe, cooker, cotton, and/or water) at least once in the prior 30 days. In the previous 6 months, $61 \%$ reported having sex with a new partner, and $40 \%$ reported having more than one partner (Table 1 ).

Most participants $(80 \%)$ reported having been tested for HIV at least once (Table 1), and of those tested, most $(82 \%)$ reported having received their test results (data not shown). Less than half $(45 \%)$ the sample reported having been aware of their HIV infection prior to enrolment. Of these, $73 \%$ had received their positive test results more than 2 years ago (data not shown); no date of diagnosis was available for 3 of the 70 participants who were previously aware of their HIV infection. No participant characteristic was significantly associated with prior diagnosis of HIV infection save marital status $\left(\chi^{2}=6.64, \quad P=0.042\right.$; Table 1).

\section{Characteristics of Participants' Reported Sex Partners}

Participants reported approximately one in five partners (19\%) to be HIV-seropositive, with most partners $(80 \%)$ communicating this information directly to the participant (data not shown). Of the partners perceived to be seronegative $(31 \%)$, participants reported asking most $(71 \%)$ whether they had been tested for HIV (data not shown). Of the partners whose serostatus was "unknown" to the participant (50\%), fewer than a third (29\%) had been asked by the participant whether they had been tested for HIV (data not shown). Participants were no more likely to report knowing the HIV status of their primary sex partners than of their other partners. Participants were more likely to perceive IDU partners as HIV-positive than as seronegative or of unknown serostatus ( $P<0.001$; data not shown). No other partner characteristic (e.g., partner's gender, partner's age, partnership type) was associated with perception of partner's serostatus.

Association Between Previous Awareness of HIV Diagnosis and Condom Use at Last Intercourse

Participants reported using condoms at last intercourse in $115(50 \%)$ of 231 partnerships (Table 2). Awareness of 
Table 1 Characteristics of HIV seropositive drug injectors, by previous diagnosis of HIV infection, St. Petersburg, Russia (2005-2008) ( $n=157$ unless otherwise noted)

\begin{tabular}{|c|c|c|c|c|c|}
\hline Characteristic & $\begin{array}{l}\text { No. }(\%) \\
\text { diagnosed }\end{array}$ & $\begin{array}{l}\text { No. }(\%) \\
\text { undiagnosed }\end{array}$ & Total & $\chi^{2}$ & $P$ \\
\hline Total & $70(45)$ & $87(55)$ & $157(100)$ & & \\
\hline \multicolumn{6}{|l|}{ Gender } \\
\hline Male & $44(63)$ & $58(67)$ & $102(65)$ & 0.247 & 0.619 \\
\hline Female & $26(37)$ & $29(33)$ & $55(35)$ & & \\
\hline $\operatorname{Age}^{\mathrm{a}}$ & $28(25,32)$ & $28(24,32)$ & $28(24,32)$ & $-0.540^{\mathrm{c}}$ & 0.590 \\
\hline \multicolumn{6}{|l|}{ Marital status } \\
\hline Married or partnered & $22(31)$ & $37(43)$ & $58(38)$ & 6.635 & 0.042 \\
\hline Single, divorced, widowed or separated & $48(69)$ & $50(57)$ & $98(62)$ & & \\
\hline \multicolumn{6}{|l|}{ Living situation } \\
\hline Alone or with partner & $24(34)$ & $22(25)$ & $46(29)$ & $0.323^{\mathrm{d}}$ & 0.315 \\
\hline With family or friends & $44(63)$ & $64(74)$ & $108(69)$ & & \\
\hline Shelter or SRO & $2(3)$ & $1(1)$ & $3(2)$ & & \\
\hline \multicolumn{6}{|l|}{ Postsecondary education } \\
\hline Yes & $39(56)$ & $49(56)$ & $88(56)$ & 0.006 & 0.939 \\
\hline No & $31(44)$ & $38(44)$ & $69(44)$ & & \\
\hline \multicolumn{6}{|l|}{ Full/part-time employment } \\
\hline Yes & $26(37)$ & $32(37)$ & $58(37)$ & 0.002 & 0.963 \\
\hline No & $44(63)$ & $55(65)$ & $99(63)$ & & \\
\hline \multicolumn{6}{|l|}{ Any legal income (past 30 days) } \\
\hline Yes & $44(66)$ & $48(65)$ & $90(92)$ & 0.009 & 0.926 \\
\hline No & $22(34)$ & $26(35)$ & $48(35)$ & & \\
\hline \multicolumn{6}{|l|}{ Ever in jail or prison } \\
\hline Yes & $34(49)$ & $32(37)$ & $66(42)$ & 2.213 & 0.137 \\
\hline No & $36(51)$ & $55(63)$ & $91(58)$ & & \\
\hline \multicolumn{6}{|l|}{ Ever tested for HIV } \\
\hline Yes & $70(100)$ & $55(63)$ & $125(80)$ & $32.338^{\mathrm{d}}$ & $<0.001$ \\
\hline No & $0(0)$ & $32(37)$ & $32(20)$ & & \\
\hline \multicolumn{6}{|l|}{ Injecting for past 9 years or longer } \\
\hline Yes & $40(57)$ & $39(45)$ & $79(50)$ & 2.353 & 0.125 \\
\hline No & $30(43)$ & $48(55)$ & $78(50)$ & & \\
\hline \multicolumn{6}{|l|}{ Injected last 30 days } \\
\hline Yes & $64(94)$ & $77(91)$ & $141(92)$ & $0.550^{\mathrm{d}}$ & 0.420 \\
\hline No & $4(6)$ & $8(9)$ & $12(8)$ & & \\
\hline \multicolumn{6}{|l|}{ Injections/month $>22^{\mathrm{b}}$} \\
\hline Yes & $29(45)$ & $42(55)$ & $71(50)$ & 1.192 & 0.275 \\
\hline No & $35(55)$ & $35(45)$ & $70(50)$ & & \\
\hline \multicolumn{6}{|l|}{ Unsafe injection 30 days $^{\mathrm{b}}$} \\
\hline Yes & $52(81)$ & $60(78)$ & $112(79)$ & 0.237 & 0.626 \\
\hline No & $12(19)$ & $17(22)$ & $29(21)$ & & \\
\hline \multicolumn{6}{|l|}{ New sex partner, 6 months } \\
\hline Yes & $43(61)$ & $52(60)$ & $95(61)$ & 0.045 & 0.833 \\
\hline No & $27(39)$ & $35(40)$ & $62(39)$ & & \\
\hline \multicolumn{6}{|l|}{ More than one sex partner, 6 months } \\
\hline Yes & $27(39)$ & $36(41)$ & $63(40)$ & 0.127 & 0.721 \\
\hline No & $43(61)$ & $51(59)$ & $94(60)$ & & \\
\hline
\end{tabular}

${ }^{\text {a }}$ Median (25th percentile, 75th percentile)

${ }^{\mathrm{b}}$ Excludes participants who did not report having injected in the past 30 days

${ }^{c} Z$-score for Wilcoxon ranksum test

d Fisher's exact Chi-square were less likely to use condoms with their primary partners (aOR 0.60, 95\% CI 0.38-0.96; $P=0.034$ ) and with IDU partners (aOR $0.39,95 \%$ CI $0.23-0.66 ; P<0.001)$. In a one's HIV infection was not associated with condom use in a model that included all partners regardless of participants' perception of partner serostatus. Instead, participants 
Table 2 Correlates of condom use at last sexual intercourse among drug injectors in St. Petersburg, Russia

\begin{tabular}{|c|c|c|c|c|c|c|c|c|}
\hline & Total & $\begin{array}{l}\text { Used condom } \\
\text { No. }(\%)\end{array}$ & uOR & $95 \% \mathrm{CI}$ & $P$-value & $\mathrm{aOR}$ & $95 \% \mathrm{CI}$ & $P$-value \\
\hline \multicolumn{9}{|c|}{ With any partner $n_{\text {participants }}=157$} \\
\hline Total partnerships & 231 & $115(50)$ & - & - & & - & - & \\
\hline \multicolumn{9}{|c|}{ Awareness of infection in positive injector } \\
\hline Yes & 106 & $52(49)$ & 1.19 & $0.64-2.20$ & 0.587 & & & \\
\hline No & 125 & $63(50)$ & Ref & & & & & \\
\hline \multicolumn{9}{|l|}{ Gender } \\
\hline Male & 152 & $81(53)$ & 1.52 & $0.80-2.90$ & 0.207 & & & \\
\hline Female & 79 & $34(43)$ & Ref & & & & & \\
\hline \multicolumn{9}{|l|}{ Age 28 plus } \\
\hline Yes & 121 & $64(53)$ & 1.41 & $0.76-2.62$ & 0.270 & & & \\
\hline No & 110 & $51(46)$ & Ref & & & & & \\
\hline \multicolumn{9}{|l|}{ Inject 9 years } \\
\hline Yes & 115 & $63(55)$ & 1.54 & $0.83-2.85$ & 0.168 & & & \\
\hline No & 116 & $52(45)$ & Ref & & & & & \\
\hline \multicolumn{9}{|c|}{ IDU's perception of partner's serostatus } \\
\hline Positive & 44 & $18(41)$ & Ref & & & & & \\
\hline Negative & 71 & $40(56)$ & 1.79 & $0.87-3.71$ & 0.114 & & & \\
\hline Unknown & 115 & $57(50)$ & 1.28 & $0.66-2.51$ & 0.466 & & & \\
\hline \multicolumn{9}{|l|}{ Partner type } \\
\hline Main & 104 & $50(48)$ & 0.67 & $0.43-1.05$ & 0.078 & 0.60 & $0.38-0.96$ & 0.034 \\
\hline Other & 100 & $61(61)$ & Ref & & & & & \\
\hline \multicolumn{9}{|c|}{ Partner injects drugs } \\
\hline Yes & 125 & $48(38)$ & 0.36 & $0.23-0.58$ & $<0.001$ & 0.39 & $0.23-0.66$ & $<0.001$ \\
\hline No & 95 & $50(63)$ & Ref & & & Ref & & \\
\hline \multicolumn{9}{|c|}{ With partners perceived to be seronegative $n_{\text {participants }}=50$} \\
\hline Total partnerships & 71 & $40(56)$ & - & - & & & & \\
\hline \multicolumn{9}{|c|}{ Awareness of infection in positive injector } \\
\hline Yes & 27 & $19(70)$ & 3.76 & $1.11-12.66$ & 0.033 & 6.68 & $1.60-27.88$ & 0.009 \\
\hline No & 44 & $20(48)$ & Ref & & & Ref & & \\
\hline \multicolumn{9}{|l|}{ Gender } \\
\hline Male & 50 & $30(60)$ & 1.75 & $0.55-5.52$ & 0.344 & & & \\
\hline Female & 21 & $10(48)$ & Ref & & & & & \\
\hline \multicolumn{9}{|l|}{ Age 28 plus } \\
\hline Yes & 33 & $20(61)$ & 1.25 & $0.44-3.58$ & 0.677 & & & \\
\hline No & 38 & $20(53)$ & Ref & & & & & \\
\hline \multicolumn{9}{|l|}{ Inject 9 years } \\
\hline Yes & 34 & $21(62)$ & 1.59 & $0.55-4.58$ & 0.391 & & & \\
\hline No & 37 & $19(51)$ & Ref & & & & & \\
\hline \multicolumn{9}{|l|}{ Partner type } \\
\hline Main & 38 & $19(50)$ & 0.52 & $0.22-1.20$ & 0.125 & & & \\
\hline Other & 29 & $20(69)$ & Ref & & & & & \\
\hline \multicolumn{9}{|l|}{ Partner injects } \\
\hline Yes & 23 & $10(43)$ & 0.42 & $0.17-1.06$ & 0.060 & 0.29 & $0.11-0.74$ & 0.009 \\
\hline No & 48 & $30(63)$ & Ref & & & Ref & & \\
\hline
\end{tabular}


Table 2 continued

\begin{tabular}{|c|c|c|c|c|c|c|c|c|}
\hline & Total & $\begin{array}{l}\text { Used condom } \\
\text { No. }(\%)\end{array}$ & uOR & $95 \% \mathrm{CI}$ & $P$-value & $\mathrm{aOR}$ & $95 \% \mathrm{CI}$ & $P$-value \\
\hline \multicolumn{9}{|c|}{ With partners of unknown status $n_{\text {participants }}=81$} \\
\hline Total partnerships & 115 & $57(50)$ & - & - & & & & \\
\hline \multicolumn{9}{|c|}{ Awareness of infection in positive injector } \\
\hline Yes & 40 & $19(48)$ & 1.01 & $0.41-2.46$ & 0.989 & & & \\
\hline No & 75 & $38(51)$ & Ref & & & & & \\
\hline \multicolumn{9}{|l|}{ Gender } \\
\hline Male & 77 & $40(52)$ & 1.67 & $0.66-4.33$ & 0.278 & & & \\
\hline Female & 38 & $17(45)$ & Ref & & & & & \\
\hline \multicolumn{9}{|l|}{ Age 28 plus } \\
\hline Yes & 61 & $34(55)$ & 1.91 & $0.79-4.58$ & 0.150 & & & \\
\hline No & 54 & $23(43)$ & Ref & & & & & \\
\hline \multicolumn{9}{|l|}{ Inject 9 years } \\
\hline Yes & 58 & $33(57)$ & 1.86 & $0.78-4.43$ & 0.162 & & & \\
\hline No & 57 & $24(42)$ & Ref & & & & & \\
\hline \multicolumn{9}{|l|}{ Partner type } \\
\hline Main & 46 & $23(50)$ & 0.76 & $0.38-1.49$ & 0.419 & & & \\
\hline Other & 56 & $32(57)$ & Ref & & & & & \\
\hline \multicolumn{9}{|l|}{ Partner injects } \\
\hline Yes & 65 & $25(38)$ & 0.32 & $0.17-0.62$ & 0.001 & 0.32 & $0.17-0.62$ & 0.001 \\
\hline No & 39 & $25(64)$ & Ref & & & Ref & & \\
\hline
\end{tabular}

separate model of partnerships limited to partners understood to be seronegative and adjusted for the strong negative correlation of condom use with partner's injection drug use, participants' awareness of their HIV infection was associated with condom use (aOR 6.68, 95\% CI 1.60-27.88; $P=0.009)$. In a model of partnerships with sex partners of unknown serostatus, participants who had been aware of their HIV infection at the time of sexual intercourse were no more likely to have used condoms than those who had been unaware. Similar to the findings for all partners, participants were less likely to use condoms with partners assumed to be an IDU (aOR 0.32, 95\% CI $0.17-0.62 ; P=0.001)$.

Prevalence and Correlates of Disclosure of Positive Status

The 70 participants who had been aware of their HIV infection prior to enrolment provided data on 106 partnerships. They reported disclosing their HIV status in $73 \%$ of these partnerships (data not shown). Participant disclosure was associated with having injected longer than 9 years (aOR 6.04, 95\% CI 1.53, 23.77; $P=0.010$ ) and with partners whom they perceived to be seropositive (aOR $45.12 ; 95 \%$ CI $2.79,730.46 ; P=0.007$ ) or IDUs (aOR
3.61; 95\% CI 1.44, 9.06; $P=0.006$ ) (data not shown). No other participant characteristic (e.g., demographic, time since diagnosis, duration of drug use, incarceration history) or partnership characteristic (e.g., partnership type, partner's age) was associated with disclosure behavior.

Association Between HIV Disclosure and Condom Use Among Participants Who Had Been Previously Aware of Their HIV Infection

Participants who were previously aware of their HIV infection used condoms at last intercourse in 52 (49\%) of 106 partnerships (Table 3). Condoms were used in a minority of encounters with partners perceived to be positive $(36 \%)$; their use was significantly more common with partners perceived to be negative $(70 \%)$ but only slightly more likely with partners whose serostatus was perceived as unknown (48\%). Disclosure was not independently associated with condom use. Instead, condom use differed only by perceived negative serostatus of partner when comparing them to partners perceived to be seropositive (aOR 3.85, 95\% CI 1.45-10.21, $P=0.007$; Table 3 ) or to all other partners (aOR 2.95, 95\% CI 1.29-6.73 $P<0.05$; data not shown). Perception of partner status was independently associated with condom use and was not confounded by any other 
Table 3 Correlates of condom use at last intercourse among drug injectors who are aware of their HIV infection, St. Petersburg, Russia

\begin{tabular}{|c|c|c|c|c|c|c|c|c|}
\hline & Total & $\begin{array}{l}\text { Used condom } \\
\text { No. }(\%)\end{array}$ & uOR & $95 \% \mathrm{CI}$ & $P$-value & $\mathrm{aOR}$ & $95 \% \mathrm{CI}$ & $P$-value \\
\hline \multicolumn{9}{|c|}{ With any partner $n_{\text {participants }}=70$} \\
\hline Total partnerships & 106 & $52(49)$ & - & - & & & & \\
\hline \multicolumn{9}{|c|}{ Disclosure of status by positive injector } \\
\hline Yes & 77 & $34(44)$ & 0.46 & $0.70-0.98$ & 0.028 & & & \\
\hline No & 29 & $18(62)$ & Ref & & & & & \\
\hline \multicolumn{9}{|l|}{ Gender } \\
\hline Male & 67 & $38(57)$ & 2.09 & $0.81-5.41$ & 0.129 & & & \\
\hline Female & 39 & $14(36)$ & Ref & & & & & \\
\hline \multicolumn{9}{|l|}{ Age $28+$} \\
\hline Yes & 69 & $32(53)$ & 1.91 & $0.75-4.85$ & 0.176 & & & \\
\hline No & 47 & $20(43)$ & Ref & & & & & \\
\hline \multicolumn{9}{|l|}{ Known +2 years } \\
\hline Yes & 77 & $39(52)$ & 1.59 & $0.55-4.58$ & 0.392 & & & \\
\hline No & 26 & $10(38)$ & Ref & & & & & \\
\hline \multicolumn{9}{|c|}{ Ever in jail or prison } \\
\hline Yes & 50 & $21(42)$ & 0.58 & $0.23-1.45$ & 0.241 & & & \\
\hline No & 56 & $31(55)$ & Ref & & & & & \\
\hline \multicolumn{9}{|l|}{ Inject 9 years +} \\
\hline Yes & 95 & $49(52)$ & 1.98 & $0.78-5.03$ & 0.150 & & & \\
\hline No & 11 & $3(27)$ & Ref & & & & & \\
\hline \multicolumn{9}{|c|}{ IDU's perception of partner's serostatus } \\
\hline Positive & 39 & $14(36)$ & Ref & & & Ref & & \\
\hline Negative & 27 & $19(70)$ & 3.85 & $1.45-10.21$ & 0.007 & 3.85 & $1.45-10.21$ & 0.007 \\
\hline Unknown & 40 & $19(48)$ & 1.60 & $0.68-3.77$ & 0.282 & 1.60 & $0.68-3.77$ & 0.282 \\
\hline \multicolumn{9}{|l|}{ Partner type } \\
\hline Main & 41 & $18(44)$ & 0.58 & $0.30-1.11$ & 0.098 & & & \\
\hline Other & 51 & $33(65)$ & Ref & & & & & \\
\hline \multicolumn{9}{|l|}{ Partner injects } \\
\hline Yes & 67 & $29(43)$ & 0.49 & $0.25-0.96$ & 0.038 & & & \\
\hline No & 33 & $19(58)$ & Ref & & & & & \\
\hline
\end{tabular}

With partners perceived to be seronegative or perceived unknown $n_{\text {participants }}=43$

\begin{tabular}{|c|c|c|c|c|c|c|c|c|}
\hline Total partnerships & 67 & $19(70)$ & - & - & & & & \\
\hline \multicolumn{9}{|c|}{ Disclosure of status by positive injector } \\
\hline Yes & 40 & $20(50)$ & 0.46 & $0.0 .19-1.12$ & 0.089 & 0.13 & $0.02-0.66$ & 0.014 \\
\hline No & 27 & $18(67)$ & Ref & & & Ref & & \\
\hline \multicolumn{9}{|l|}{ Gender } \\
\hline Male & 46 & $30(65)$ & 3.10 & $0.85-11.28$ & 0.086 & 23.61 & $1.59-349.97$ & 0.022 \\
\hline Female & 21 & $8(38)$ & Ref & & & Ref & & \\
\hline \multicolumn{9}{|l|}{ Age $28+$} \\
\hline Yes & 35 & $24(69)$ & 2.52 & $0.79-8.03$ & 0.040 & & & \\
\hline No & 32 & $14(44)$ & Ref & & & & & \\
\hline \multicolumn{9}{|l|}{ Known +2 years } \\
\hline Yes & 49 & $29(49)$ & 2.41 & $0.60-9.71$ & 0.217 & & & \\
\hline No & 15 & $6(40)$ & Ref & & & & & \\
\hline \multicolumn{9}{|l|}{ Ever in jail or prison } \\
\hline Yes & 31 & $14(45)$ & 0.44 & $0.14-1.42$ & 0.173 & 0.02 & $0.0-0.30$ & 0.005 \\
\hline No & 36 & $24(67)$ & Ref & & & & & \\
\hline
\end{tabular}


Table 3 continued

\begin{tabular}{|c|c|c|c|c|c|c|c|c|}
\hline & Total & $\begin{array}{l}\text { Used condom } \\
\text { No. }(\%)\end{array}$ & $\mathrm{uOR}$ & $95 \% \mathrm{CI}$ & $P$-value & $\mathrm{aOR}$ & $95 \% \mathrm{CI}$ & $P$-value \\
\hline \multicolumn{9}{|c|}{ Inject 9 years +} \\
\hline Yes & 37 & $25(68)$ & 2.19 & $0.69-6.95$ & 0.182 & 25.95 & $2.65-253.81$ & 0.005 \\
\hline No & 30 & $13(43)$ & Ref & & & & & \\
\hline \multicolumn{9}{|c|}{ IDU's perception of partner's serostatus } \\
\hline Negative & 27 & $19(70)$ & 2.61 & $1.00-6.77$ & 0.049 & 8.65 & $1.67-44.65$ & 0.010 \\
\hline Unknown & 40 & $19(48)$ & Ref & & & & & \\
\hline \multicolumn{9}{|l|}{ Partner type } \\
\hline Main & 25 & $12(48)$ & 0.61 & $0.26-1.42$ & 0.249 & & & \\
\hline Other & 37 & $25(68)$ & Ref & & & & & \\
\hline \multicolumn{9}{|c|}{ Partner injects } \\
\hline Yes & 34 & $18(53)$ & 0.60 & $0.26-1.41$ & 0.244 & & & \\
\hline No & 27 & $16(59)$ & Ref & & & & & \\
\hline
\end{tabular}

variable. In a model limited to partners perceived to be susceptible to HIV infection (i.e., perceived to be seronegative or of unknown serostatus), disclosure was independently and negatively associated with condom use (aOR 0.13 , 95\% CI $0.02-0.66 ; P=0.014)$. In the same model there was a positive and independent association between condom use and male gender of participant (aOR 23.61, 95\% CI $1.59-349.97, P=0.022)$. In addition, condoms were more likely to be used with partner of perceived negative serostatus compared to partner of unknown serostatus (aOR $8.65,95 \%$ CI $1.67-44.65, P=0.010$ ).

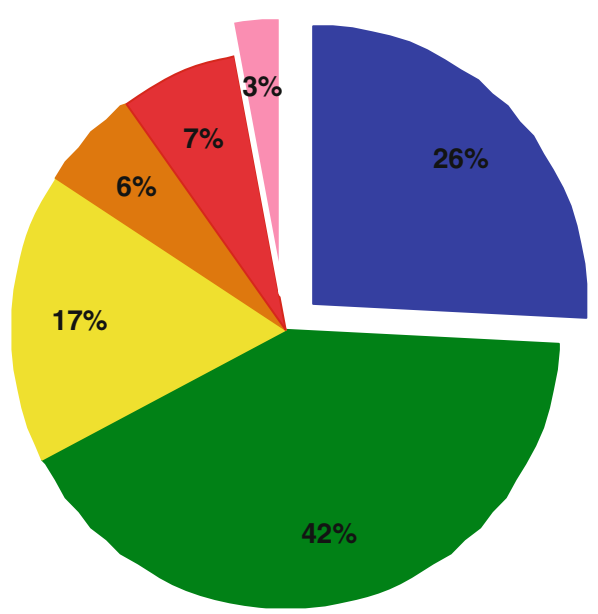

Fig. 2 Unprotected sex between HIV-seropositive participants and partners who were vulnerable to HIV infection $(n=89)$. Of the 89 unprotected sexual encounters between HIV-seropositive participants and partners perceived to be either seronegative or of unknown serostatus, 23 (26\%) occurred between participants unaware of their HIV infection and seronegative partners, $37(42 \%)$ between participants unaware of their infection and a partner of unknown serostatus, 5 (6\%) between participants who knew of their infection and had disclosed to the seronegative partner, $3(3 \%)$ between participants
Potential for HIV Transmission in Unprotected Serodiscordant Partnerships

We calculated the number of partnerships with the potential for HIV transmission, that is, unprotected sex partnerships between HIV-seropositive participants and partners perceived to be either seronegative or of unknown serostatus $(n=89)$. The vast majority of these were unprotected vaginal sex, with only four encounters noting the occurrence of unprotected anal sex. Among these, partnerships in which participants had been unaware of

Unaware, Partner HIV Negative

Unaware, Partner HIV Unknown

Aware, Partner HIV Unknown, Disclosed

Aware, Partner HIV Negative, Disclosed

Aware, Partner HIV Unknown, Did Not Disclose

Aware, Partner HIV Negative, Did Not Disclose

who knew of their infection and seronegative partners but without disclosure, $6(7 \%)$ between participants who knew of their infection and partners of unknown status but without disclosure, and $15(17 \%)$ between participants who knew of their infection and had disclosed to the partner of unknown serostatus. Hence, there were only approximately $3 \%$ of unprotected sexual encounters in which disclosure of serostatus may have influenced the decision to use a condom. (Color figure online) 
their HIV infection and understood their partner to be HIVnegative comprised 26\% $(n=23)$, and partnerships in which participants had been aware of their infection but had not disclosed to their seronegative partners comprised $3 \%(n=3$; Fig. 2$)$.

\section{Discussion}

The study results indicate that mere awareness of one's own HIV infection is not associated with condom use. Rather, among IDUs who were aware of their HIV infection, partner's perceived serostatus was the strongest predictor of condom use at last intercourse. Compared to partnerships with partners perceived to be positive or of unknown serostatus, partnerships between IDUs who were aware of their infection and partners perceived to be seronegative were characterized by a relative lack of disclosure but more condom use. Consistent with other studies [40, 53, 54], we found that disclosure was not associated with increased condom use. Instead, disclosure to potentially negative partners was associated with less condom use.

Among participants who were aware of their HIV infection at time of last intercourse, disclosure was independently associated with (1) the perception that their sex partner was also HIV-positive, (2) the perception that their partner was also an IDU, and (3) the participant having injected for at least 9 years. The first finding is consistent with other studies' findings that disclosure was less likely when partners' serostatus was either seronegative or unknown [28, 38-40]. Disclosure to partners who were perceived to be fellow injectors may be related to the first finding and reflects the harsh reality of HIV in Russia: that anyone who injects drugs is likely to become infected. The fact that no other personal or partner characteristics or partnership type were associated with disclosure contrasts with studies of disclosure practices among US populations [22, 26-33, 37, 43, 44, 55]. Further research is warranted to confirm or refute our findings.

Among HIV-positive IDUs who were aware of their infection, it appears that the perception of partners as uninfected, more than the act of disclosure, was associated with condom use. Potential exposure of perceived negative partners was much more common among participants who had been unaware of their HIV infection at the time of last sexual contact. We applied the adjusted odds ratio between prior diagnosis of HIV infection and condom use with partners perceived to be negative given in Table 2 (aOR: 6.68, CI: 1.60-27.88) and the proportion of partnerships in which an undiagnosed participant with HIV infection had exposed potentially uninfected partners $(0.26$, binomial $\mathrm{CI}$ : $0.17-0.36$ ) to a standard formula for population attributable risk percent (PAR\%).
$\operatorname{PAR} \%=P_{\text {exposed }}(\mathrm{RR}-1) /\left[1+P_{\text {exposed }}(\mathrm{RR}-1)\right]$

This suggests that if half of the undiagnosed HIV-infected IDUs from the sampling frame were to learn of their HIV infection, the number of potentially uninfected partners being exposed by infected injectors would be reduced by $60 \%$ (CI 9-90\%). Conversely, disclosure of HIV infection to potentially uninfected partners was associated with a somewhat lesser likelihood of using condoms. It is of interest that males who were aware of their HIV infection were significantly more likely than females to report having used a condom at time of last intercourse. This may be due to the fact that, compared to females, males can more easily opt to use a condom with minimal need for condom negotiation and no need for disclosure.

Limitations of the study included the inherent potential biases associated with self-reported data such as recall bias and social desirability. Due to the relatively small sample size, there was insufficient power to detect effect modification; under these conditions, stratification was considered to be the appropriate analytic approach. The cross-sectional nature of this study does not permit determination of causal associations. The survey questioned participants about whether they had asked their partners if they had been tested, but (1) did not follow up to ask whether partners had responded to the question or what those test results were and (2) whether discussions of serostatus happened before or after initiation of the sexual partnership. These methodological problems have been noted elsewhere [27]. We therefore cannot ascertain the degree to which a recent negative test is associated with protected sex with HIVpositive partners who are aware of their infection. Detailed data about the timing of disclosure relative to the onset of sexual relations should be collected in future studies. Further research is needed to understand the process by which individuals ascertain their partners' HIV serostatus and to identify those factors which enter into condom use decisions. Finally, the study from which the data for this analysis were taken did not inquire whether HIV-diagnosed participants had been declined by potential sexual partners due to participants' disclosure of HIV-positive status; while taking such scenarios into account would not change the proportion of partnerships in which condoms were used, it would impact the proportion of unprotected partnerships occurring post-disclosure.

Evidence from studies outside of Russia suggests that sexual risk behaviors may contribute more than injection risk behaviors to HIV transmission among IDUs when injection risks are reduced through educational, behavioral, or structural interventions [56, 57], but few interventions specifically target the reduction of sexual risk among this population [58]. Our experience working with this population in St. Petersburg indicates that condoms are readily 
available and inexpensive at most convenience stores, pharmacies, and kiosks; they are also free at syringe exchanges and outreach programs. There are few published reports of condom use rates among IDUs or even within the general population of Russia, and even less is known about these rates among partner types (e.g., new, main, casual partners). However, there is some evidence that condom use rates are generally low, with $45 \%$ of injectors in one study reporting no condom use in the previous 6 months [59], another study noting consistent condom use for only $44 \%$ of a sample of Moscow youths [60], and 19-25\% of respondents in a representative probability sample of Russian households reporting condom use at their last sexual encounter [61]. Elsewhere in the world the rates of unprotected sex for IDUs ranged from 28 to 77\% [62-68]. Our findings suggest the need for interventions targeting increased condom use among Russian IDUs and that HIV testing - and provision of test results—should be encouraged among all members of social networks in which HIV is prevalent. In our analysis fewer than half the participants were aware of their infection, but when aware, condom use was significantly more likely to occur with partners perceived to be seronegative. These findings, compounded with the negative association between condom use and disclosure, suggest that public health benefit (in terms of decreasing sexual exposure to HIV by uninfected partners) may accrue from the scaling up HIV testing services and interventions that increase the likelihood of individuals receiving their test results.

Acknowledgments Support for this work was provided by NIDA grants U01DA017373, William Zule, RTI International; U01DA 017377, Martin Iguchi and Sandra Berry, RAND Corporation; U01DA017378, Lawrence Ouellet, University of Illinois, Chicago; U01DA017387, Robert Heimer and Andrei Kozlov, Yale University and the Biomedical Center; U01DA017394, Steve Shoptaw and Pamina Gorbach, University of California, Los Angeles. Data in St. Petersburg was collected by the Biomedical Center in collaboration with Yale University as part of SATH-CAP Study ("Doverie").

Open Access This article is distributed under the terms of the Creative Commons Attribution Noncommercial License which permits any noncommercial use, distribution, and reproduction in any medium, provided the original author(s) and source are credited.

\section{References}

1. Elovich R, Drucker E. On drug treatment and social control: Russian narcology's great leap backwards. Harm Reduct J. 2008;5:23.

2. UNAIDS. 2006 Report of the global AIDS epidemic. 2007. Available at www.unaids.org/en/HIV_data/2006GlobalReport/default.asp . Accessed 15 Nov 2007.

3. Goliusov AT, Dementyeva LA, Ladnaya NN, et al. Country progress report of the Russian federation on the implementation of the declaration of commitment on HIV/AIDS. Moscow, Federal Service for Surveillance of Consumer Rights Protection and Human Well-Being of the Russian Federation. 2009. Available at http://data.unaids.org/pub/Report/2008/russia_2008_country_progress_report_en.pdf. Accessed 21 May 2009.

4. UNAIDS. AIDS epidemic regional update, Eastern Europe and Central Asia. 2007. Available at http://data.unaids.org/pub/Report/ 2008/jc1529_epibriefs_eeurope_casia_en.pdf. Accessed 21 May 2009.

5. Kozlov AP. HIV in Russia, Belorussia, and Ukraine. Russ J HIV/ AIDS Relat Probl. 2000;4:11-4.

6. Krupitsky EM, Zvartau EE, Lioznov DA, et al. Co-morbidity of infectious and addictive diseases in St. Petersburg and the Leningrad Region. Russ Eur Addict Res. 2006;12(1):12-9.

7. Semaille C, Alix J, Downs AM, Hamers FF. The HIV infection in Europe: large East-West disparity. Euro Surveill. 2003;8(3): $57-64$.

8. United Nations Development Programme. HIV/AIDS in Eastern Europe and the commonwealth of independent states: reversing the epidemic. Facts and policy options. 2007. Available at http://europeandcis.undp/hiv/files/full_English.pdf. Accessed 15 Nov 2007.

9. Abdala N, Carney JM, Durante AJ, et al. Estimating the prevalence of syringe-borne and sexually transmitted diseases among injection drug users in St. Petersburg, Russia. Int J STD AIDS. 2003;14:697-703.

10. Niccolai LM, Shcherbakova IS, Toussova OV, Kozlov AP, Heimer R. The potential for bridging of HIV transmission in the Russian Federation: sex risk behaviors and HIV prevalence among drug users (DUs) and their non-DU sex partners. J Urban Health. 2009;86(Suppl 1):131-43.

11. Ostrovsky D. Injection drug use, HIV, hepatitis, and overdose in St. Petersburg. In: 8th International conference on AIDS, cancer, and related problems. St. Petersburg, Russia; 2000.

12. Ostrovsky DV. WHO drug injecting study in St. Petersburg, phase two. In: XIV International AIDS conference, Barcelona, Spain; 2002 [abstract WePeC6081].

13. Shaboltas AV, Toussova OV, Hoffman IF, et al. HIV prevalence, sociodemographic, and behavioral correlates and recruitment methods among injection drug users in St. Petersburg, Russia. J Acquir Immune Defic Syndr. 2006;41:657-63.

14. Smol'skaia TT, Khodakevich LN, Kobyshcha IuV, et al. An HIV prevalence study by means of sentinel epidemiological surveillance among injection narcotic abusers in Saint Petersburg [Russian]. Zh Mikrobiol Epidemiol Immunobiol. 2000;4:31-3.

15. Burchell AN, Calzavara LM, Orekhovsky V, Ladnaya NN. Canada AIDS Russia project, Russian HIV response network. Characterization of an emerging heterosexual HIV epidemic in Russia. Sex Transm Dis. 2008;35:807-13.

16. Hamers FF, Downs AM. HIV in Central and Eastern Europe. Lancet. 2003;361:1035-44.

17. United Nations Programme on HIV/AIDS (UNAIDS). AIDS epidemic update: December 2003. 2009. Available at http://www.who. int/hiv/pub/epidemiology/epi2003/en/. Accessed 18 Mar 2009.

18. Centers of Disease Control and Prevention. Advancing HIV prevention: new strategies for a changing epidemic-United States, 2003. MMWR Morb Mortal Wkly Rep. 2003;52:329-32.

19. Janssen RS, Valdisserri RO. HIV prevention in the United States: increasing emphasis on working with those living with HIV. J Acquir Immune Defic Syndr. 2004;37(Suppl 2):S119-21.

20. Simoni JM, Mason HR, Marks G, Ruiz MS, Richardson JL. Women living with HIV: sexual behaviors and counseling experiences. Women Health. 1995;23:17-26.

21. Sowell RL, Lowenstein A, Moneyham L, Demi A, Mizuno Y, Seals B. Resources, stigma, and patterns of disclosure in rural women with HIV infection. Public Health Nurs. 1997;14:302-12. 
22. Stein MD, Freedberg KA, Sullivan LM, et al. Sexual ethics: disclosure of HIV-positive status to partners. Arch Int Med. 1998; 158:253-7.

23. Amirkhanian YA, Kelly JA, Issayev DD. AIDS knowledge, attitudes, and behaviour in Russia: results of a population-based, random-digit telephone survey in St. Petersburg. Int J STD AIDS. 2001;12:50-7.

24. Balabanova Y, Coker R, Atun RA, Drobniewski F. Stigma and HIV infection in Russia. AIDS Care. 2006;18:846-52.

25. Niccolai LM, Toussova OV, Verevochkin SV, Barbour R, Heimer R, Kozlov AP. High HIV prevalence, suboptimal HIV testing, and low knowledge of HIV-positive serostatus among injection drug users in St. Petersburg, Russia. AIDS Behav. 2010;14(4):932-41.

26. Latka MH, Mizuno Y, YingFeng W, et al. Are feelings of responsibility to limit the sexual transmission of HIV associated with safer sex among HIV-positive injection drug users? J Acquir Immune Defic Synd. 2007;46(Supp 2):S88-95.

27. Niccolai LM, Dorst D, Myers L, Kissinger PJ. Disclosure of HIV status to sexual partners: predictors and temporal patterns. Sex Transm Dis. 1999;26:281-5.

28. DeRosa CJ, Marks G. Preventive counseling of HIV-positive men and self-disclosure of serostatus to sex partners: new opportunities for prevention. Health Psychol. 1998;17:224-31.

29. Knight KR, Purcell D, Dawson-Rose C, Halkitis PN, Gomez CA, Seropositive Urban Injectors Study Team. Sexual risk taking among HIV-positive injection drug users: contexts, characteristics, and implications for prevention. AIDS Educ Prev. 2005;17(Suppl A):76-88.

30. Perry SW, Card CA, Moffat M, Ashman R, Fishman B, Jacobsberg LB. Self-disclosure of HIV infection to sexual partners after repeated counseling. AIDS Educ Prev. 1994;6(5):403-11.

31. Semple SJ, Patterson TL, Grant I. Psychosocial characteristics and sexual risk behavior of HIV + men who have anonymous sex partners. Psychol Health. 2004;19(1):71-87.

32. Semple SJ, Patterson TL, Shaw WS, Pedlow CT, Grant I. Disclosure of HIV seropositivity to sexual partners: an application of social cognitive theory. Behav Ther. 1999;30:223-37.

33. Wolitski RJ, Bailey CJ, O’Leary A, Gomez CA, Parsons JT. Seropositive urban men's study (SUMS). Self-perceived responsibility of HIV-seropositive men who have sex with men for preventing HIV transmission. AIDS Behav. 2003;7(4):363-72.

34. Anderson RM, May RM. Epidemiologic parameters of HIV transmission. Nature. 1988;333:514-9.

35. Garnett GP, Anderson RM. Contact tracing and the estimation of sexual mixing patterns: the epidemiology of gonococcal infections. Sex Transm Dis. 1993;20:181-91.

36. Gupta S, Anderson RM, May RM. Networks of sexual contact: implications for the pattern of spread of HIV. AIDS. 1989;3:807-17.

37. Serovich JM. A test of two HIV disclosure theories. AIDS Educ Prev. 2001;13(4):355-64.

38. Ciccarone D, Kanouse D, Collins R, et al. Sex without disclosure of positive HIV serostatus in a US probability sample of persons receiving medical care for HIV infection. Am J Public Health. 2003;93(6):949-54.

39. Marks G, Crepaz N. HIV-positive men's sexual practices in the context of self-disclosure of HIV status. J Acquir Immune Defic Syndr. 2001;7:866-9.

40. Parsons JT, Missildine W, van Ora J, Purcell DW, Gomez CA. HIV serostatus disclosure to sexual partners among HIV-positive injection drug users. AIDS Patient Care STDS. 2004;18:457-69.

41. Derlega VJ, Winstead BA, Oldfield EC III, Barbee AP. Close relationships and social support in coping with HIV: a test of sensitive interaction systems theory. AIDS Behav. 2003;7(2): 119-29.
42. Zea MC, Reisen CA, Poppen PJ, Diaz RM. Asking and telling: communication about HIV status among Latino HIV-positive gay men. AIDS Behav. 2003;7(2):143-52.

43. Cusick L, Rhodes T. The process of disclosing positive HIV status: findings from qualitative research. Cult Health Sex. 1999;1(1):3-18.

44. Klitzman RL. Self-disclosure of HIV status to sexual partners: a qualitative study of issues faced by gay men. J Gay Lesbian Med Assoc. 1999;3(2):39-49.

45. Yoshioka MR, Schustack A. Disclosure of HIV status: cultural issues of Asian patients. AIDS Patient Care STDS. 2001;15(2): $77-82$.

46. Realo A, Goodwin R. Family-related allocentrism and HIV risk behavior in Central and Eastern Europe. J Cross Cult Psychol. 2003;34(6):690-701.

47. Parsons JT, VanOra J, Missildine W, Purcell DW, Gomez CA. Positive and negative consequences of HIV disclosure among seropositive injection drug users. AIDS Educ Prev. 2004;16: 459-75.

48. Schlumberger MG, Desenclos J-C, Papaevangelou G, Richardson SC, Ancelle-Park R, European Community Study Group on HIV in Injecting Drug Users. Knowledge of HIV serostatus and preventive behaviour among European injecting drug users: second study. Eur J Epidemiol. 1999;15:207-15.

49. Iguchi MY, Ober AJ, Berry S, et al. Simultaneous recruitment of drug users and men who have sex with men in the United States and Russia using respondent driven sampling: sampling methods and implications. J Urban Health. 2009;86(Suppl 1):5-31.

50. Heckathorn DD. Respondent-driven sampling: a new approach to the study of hidden populations. Soc Probl. 1997;44:174-99.

51. Heckathorn DD. Extensions of respondent-driven sampling: analyzing continuous variables and controlling for different recruitment. Sociol Methodol. 2007;37:151-208.

52. StataCorp. Stata statistical software: release 10. 10 ed. College Station, TX: StataCorp; 2007.

53. Dave SS, Stephenson J, Mercey DE, Panahmand N, Jungmann E. Sexual behavior, condom use, and disclosure of HIV status in HIV infected heterosexual individuals attending an inner London HIV clinic. Sexy Transm Infect. 2006;82:117-20.

54. Wolitski R, Rietmeijer CA, Goldbaun GM, Wilson RM. HIV serostatus disclosure among gay and bisexual men in four American cities: general patterns and relation to sexual practices. AIDS Care. 1998;10(5):599-610.

55. Kalichman SC, Nachimson D. Self-efficacy and disclosure of HIV-positive serostatus to sex partners. Health Psychol. 1999;18(3):281-7.

56. Kral AH, Bluthenthal RN, Lorvick J, Gee L, Baccetti P, Edlin BR. Sexual transmission of HIV-1 among injection drug users in San Francisco, USA: rick-factor analysis. Lancet. 2001; 357(9266):1397-401.

57. Strathdee SA, Galai N, Safaiean M, et al. Sex differences in risk factors for HIV seroconversion among injection drug users: a 10year perspective. Arch Intern Med. 2001;161(10):1281-8.

58. Semaan S, Des Jarlais DC, Sogolow E, et al. A meta-analysis of the effect of HIV prevention interventions on the sex behaviors of drug users in the United States. J Acquir Immune Defic Syndr. 2002;30(Suppl 1):S73-93.

59. Raj A, Cheng DM, Krupitsky EM, Coleman S, Bridden C, Samet $\mathrm{JH}$. Correlates of any condom use among Russian narcology patients reporting recent unprotected sex. AIDS Behav. 2009;13: $210-7$.

60. Bobrova N, Sergeev O, Grechukhina T, Kapiga S. Social-cognitive predictors of consistent condom use among young people in Moscow. Perspect Sex Reprod. 2005;37:174-8.

61. Gerber TP, Berman D. Heterogeneous condom use in contemporary Russia. Stud Fam Plann. 2008;39:1-17. 
62. Anderson JE, Cheney R, Clatts M, Faruque S, Kipke M, Long A, et al. HIV risk behavior, street outreach, and condom use in eight high-risk populations. AIDS Educ Prev. 1996;8:191-204.

63. Belanger D, Godin G, Alary M, Bernard P-M. Factors explaining the intention to use condom among injecting drug users participating in a needle-exchange program. J Appl Soc Psychol. 2002;32:1047-63.

64. Bogart LM, Kral AH, Scott A, Anderson R, Flynn N, Gilbert ML, et al. Condom attitudes and behaviors among injection drug users participating in California syringe exchange programs. AIDS Behav. 2005;9:423-32.

65. Booth RE, Kwiatkowski CF, Chitwood DD. Sex related HIV risk behaviors: differential risks among injection drug users, crack smokers, and injection drug users who smoke crack. Drug Alcohol Depend. 2000;58:219-26.

66. Shafer KP, Hahn JA, Lum PJ, Ochoa K, Graves A, Moss A. Prevalence and correlates of HIV infection among young injection drug users in San Francisco. J Acquir Immune Defic Syndr. 2002;31:422-31.

67. Vanichseni S, Des Jarlais DC, Choopanya K, Friedmann P, Wenston J, Sonchai $\mathrm{W}$, et al. Condom use with primary partners among injecting drug users in Bangkok, Thailand and New York City, United States. AIDS. 1993;7:887-91.

68. White D, Phillips K, Mulleady G, Cupitt C. Sexual issues and condom use among injecting drug users. AIDS Care. 1993; 5:427-37. 